\title{
Modified hybrid fixator for high-energy Schatzker V and VI tibial plateau fractures
}

\author{
Hisam Muhamad Ariffin • Nidzwani M. Mahdi • \\ Shaharuddin A. Rhani • Azmi Baharudin • \\ Mohamad Hassan Shukur
}

Received: 11 November 2009/ Accepted: 24 January 2011/Published online: 11 February 2011

(C) The Author(s) 2011. This article is published with open access at Springerlink.com

\begin{abstract}
High-energy tibial plateau fractures associated with severe soft tissue injury are difficult to manage. The risk of wound complications following open reduction and internal fixation is notably high owing to extensive soft tissue dissection. Alternatively, application of hybrid external fixator minimizes soft tissue dissection and provides adequate fracture stabilization to allow early range of motion and correction of any mal-alignment. With this technique, soft tissue complications particularly surgical site infections are expected to be significantly reduced. This prospective study aims to determine the effectiveness of a modified hybrid external fixator in the management of high-energy tibial plateau fractures. Thirty-three patients with high-energy Schatzker V and VI tibial plateau fracture with severe soft tissue injury precluding formal open reduction were enrolled into the study. The fixator was a construct combining the Ilizarov ring with a monolateral external fixator. The results-bony union, range of motion, and associated complications of the treatment-were assessed. All fractures united within an average time of 14 weeks. Neither loss of reduction nor surgical site wound breakdown/osteomyelitis was noted. Eight patients developed superficial pin track infection and one septic arthritis of the knee joint. Hybrid external fixation is a safe option for complex high-energy tibial plateau fractures by simultaneously providing adequate fracture stabilization and protection of soft tissue healing to achieve bony union. The complication is mainly related to pin tract infection.
\end{abstract}

H. M. Ariffin $(\bowtie) \cdot$ N. M. Mahdi · S. A. Rhani · A. Baharudin - M. H. Shukur

Faculty of Medicine, Universiti Kebangsaan

Malaysia Medical Centre, Kuala Lumpur, Malaysia

e-mail: hesam8791@yahoo.com
Keywords High-energy tibial plateau fractures . Severe soft tissue injury $\cdot$ Hybrid external fixator

\section{Introduction}

High-energy tibial plateau fractures of Schatzker V and IV are complex fractures often associated with severe soft tissue injury and high risk of wound complications following formal open reduction and internal fixation [1]. Treatment difficulty is further compounded by problem of choosing the stabilization option to provide a stable fixation of a commonly comminuted fracture. Although dual plating is biomechanically proven to be the best stabilization option, it requires extensive soft tissue dissection with a potential high rate of post-operative complications particularly when single midline incision was used for dual plating [1-4].

Hybrid external fixation has recently been advocated. Early experiences appear to indicate its effectiveness in the treatment of these difficult fractures [2, 5-8]. It relies on the periarticular ring construct for stable fixation of the tibial condyles and monolateral fixator for fixation on the shaft to provide a stable fixation block. It has been shown to provide adequate stability to maintain fracture alignment without the need of massive soft tissue dissection, and hence minimizing the potential soft tissue complications. The construct allows early joint mobilization, allowing cartilage regeneration and joint remodeling, which decreases the risk of joint stiffness.

The commercially available hybrid external fixators are expensive, and the need to keep using these fixators on patients for a prolonged period has raised an issue related to its cost-effectiveness. This has lead us to innovate a cheaper and readily available hybrid external fixator 
construct by combining an Ilizarov ring with monolateral external fixator system (Figs. 1, 2). We have been using this modified version of hybrid external fixation for the treatment of complex tibial plateau fractures since 2002 . This study highlights the technique and results of treatment using this modified hybrid external fixation for such injuries.

\section{Materials and method}

The indication for this method of treatment was Schatzker V and VI tibial plateau fractures with associated severe soft tissue injury of Tscherne and Oestern grade 2 and 3. In our institution, this method of treatment was reserved for the most severe fractures of the tibial plateau. Patients with fracture associated with vascular injury and those judged to be unable to take care of the fixator as the method requires patient's cooperation in pin site care and dressing were excluded.

This prospective study was conducted from July 2003 till December 2007. Thirty-three patients with complex tibial plateau fracture were enrolled. Of 31 patients available for follow-up, 26 were men and 5 women. All injuries were due to motor vehicle accidents. There were 19 Schatzker-V and 12 Schatzker-VI injuries. Gustillo and Anderson open fracture of grade-IIIA was encountered in 13 patients, and 18 patients had severe closed soft tissue injuries. There were 10 cases of compartment syndrome. The patients diagnosed with compartment syndrome based on clinical examination undergo an emergency fasciotomy

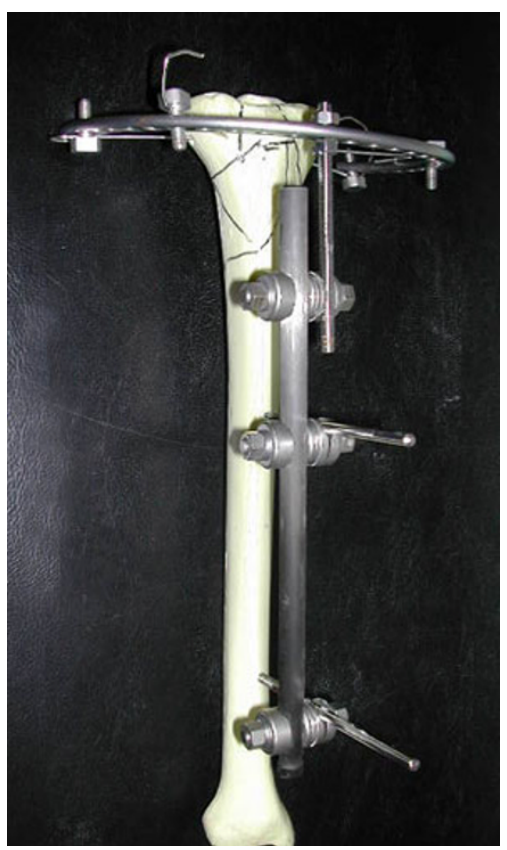

Fig. 1 Modified hybrid external fixator

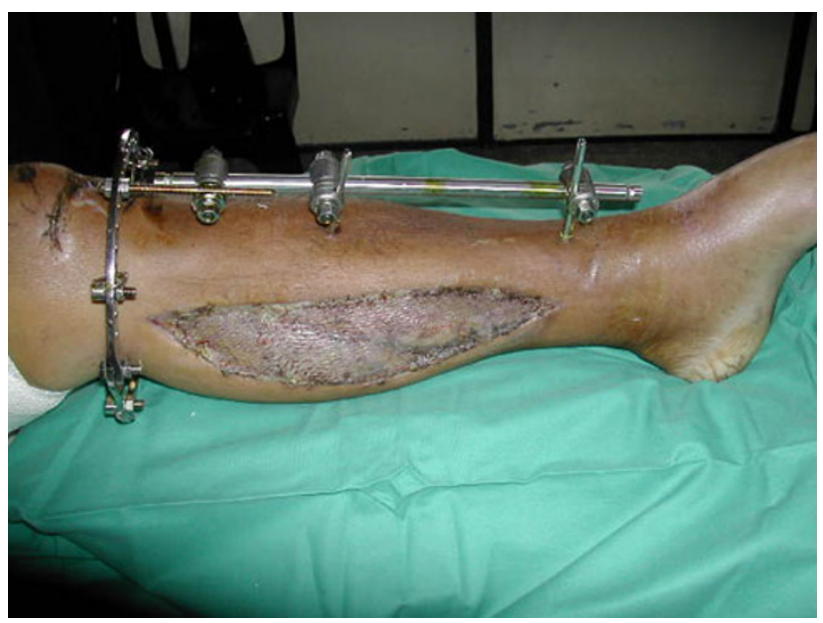

Fig. 2 Clinical application

followed by reduction and application of the hybrid fixator under emergency.

The operation was performed under spinal or general anesthesia. Radiolucent operating table and fluoroscopy were used to ease the operation. The fragments were aligned by simple manual traction by an assistant. The condylar fragments were compressed with a large tenaculum forceps or a pelvic reduction forceps and fixed with 7.0-mm cannulated screws or the long 3.5-mm screws from the pelvic sets depending on the size of the fragment when possible. Failure to reduce the articular fragments or the presence of articular depression often necessitates an open reduction through a small anteromedial or an anterolateral approach to elevate the articular surface using the blunt end of the metallic Yankaeur suction tip (Fig. 3). Two 1.8-mm wires were introduced in the safe zone, $15 \mathrm{~mm}$ from the joint line [9]. The minimal angle in between the two oblique wires is $60^{\circ}$ respecting the anatomic constraints. The ring is attached and the wires were tensioned. The meta-diaphyseal alignment was corrected, and two Schanz pins were inserted at the diaphysis. The Shantz pins were connected to the external fixator bar, which was then coupled to the ring using the external fixator clamp (Fig. 1). The alignment was again checked under fluoroscopy in both the standard anteroposterior and the lateral planes. The fracture was reduced by closed means in 11 patients. Bone grafting was not used at all. All patients were instructed on fixator care and taught to do daily pin sites cleansing with hydrogen peroxide. All patients were started on passive range of motion exercise on the third post-operative day and active motion by 1 week. The patient was started on non-weight-bearing 6-8 weeks, and partial weight-bearing ambulation is allowed depending on the amount of callus formation after that. Full weightbearing were allowed after removal of the fixator. Serial standing radiographic examination of the knee and the tibia 
Fig. 3 Steps and techniques of articular reduction

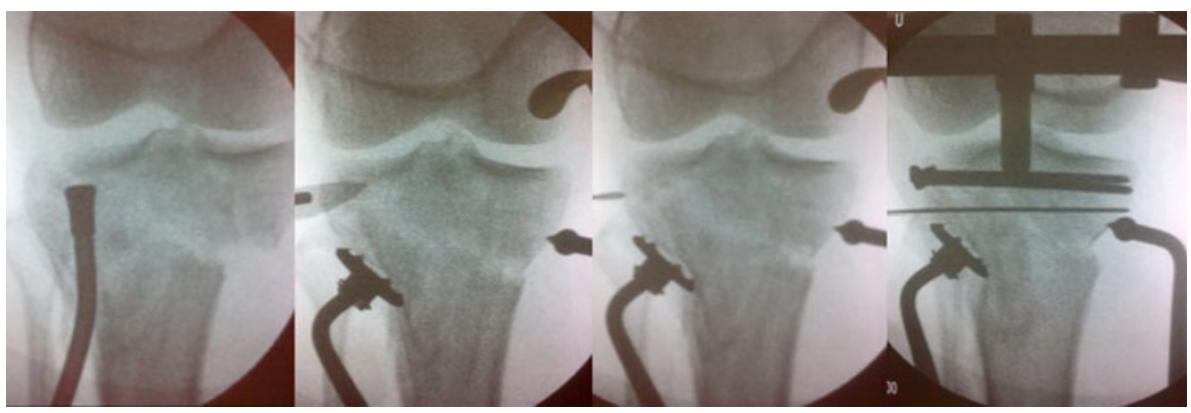

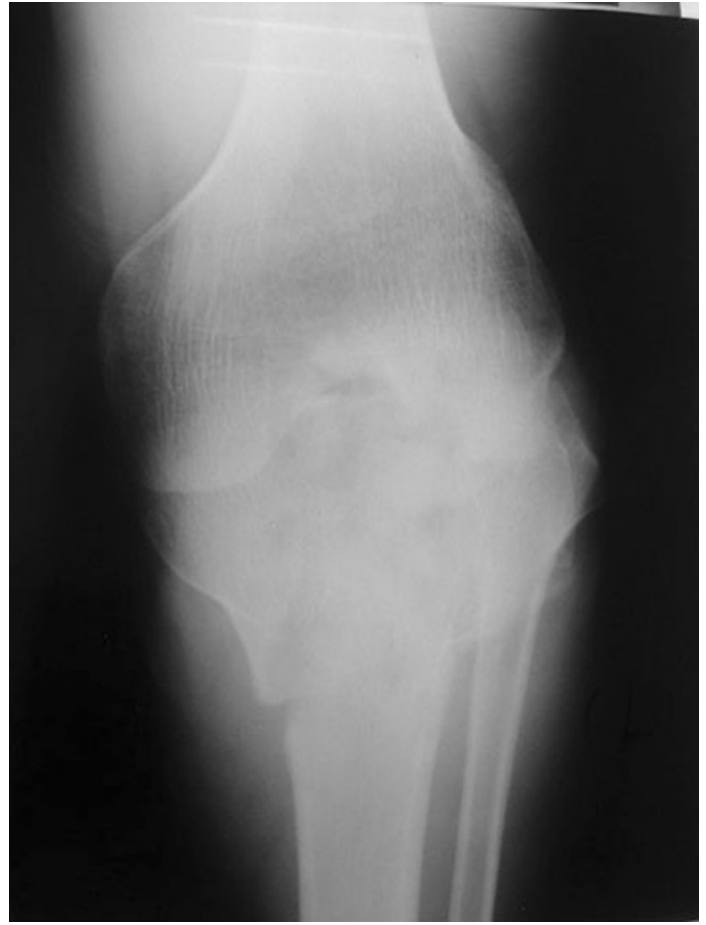

Fig. 4 X-ray of Schatzker-VIIV fracture with patella fracture AP view

in $\mathrm{AP}$ and lateral planes was performed at 6 weeks, 3 months, 6 months, and 1 year post-operatively.

During follow-up, two members of the study group assess the patients in terms of range of motion, fracture union clinically and radiologically (Figs. 4, 5, 6, 7, 8, 9), any loss of reduction radiologically, any hardware-related complications like wire breakage, clamp failures, surgical sites infections, osteomyelitis, and complications related to the surgery like nerve injury from inadvertent pin placement. Functional score according to Rassmussen [10] was assessed at 6 months and 1 year.

\section{Results}

The mean patients' age at the time of injury was 44 years (range 21-68 years). The average duration of hospitalization

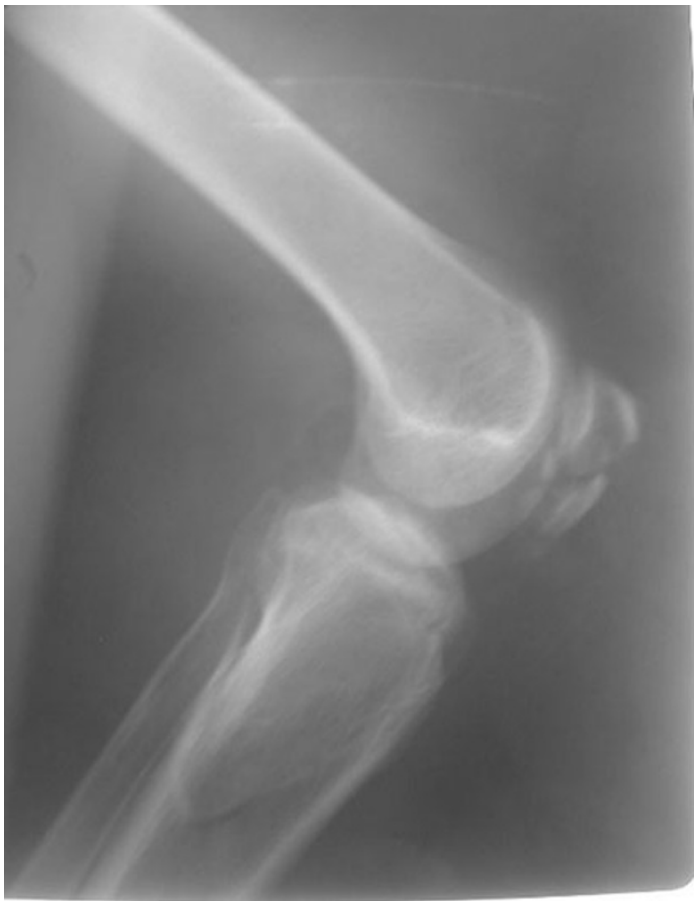

Fig. 5 X-ray of Schatzker-VIIV fracture with patella fracture lateral view

was 32 days (range 6-57 days). All fractures united in an average time of 14 weeks (range $8-20$ weeks). Two fractures have varus malunion (one with $12^{\circ}$ (Rasmussen knee score of 21) and another with $10^{\circ}$ of varus (Rasmussen knee score of 24)).

The mean duration of fixator usage was 14 weeks (range 6-20 weeks). The fixator was prematurely removed at 6 week due to clamp failure with subsequent loosening of the wire. Hinged cast bracing was sequentially used in two patients (one for a Parkinsonism patient and another for a proximal wire loosening due to clamp failure requiring early removal of fixator). A patella tendon bearing cast was used in a patient who had a concomitant ipsilateral openIIIB midshaft tibia fracture that united at 6 month.

Eight patients developed superficial pin track infection at the wires over the proximal ring. The infection was controlled with local pin site care and oral Cloxacillin. 


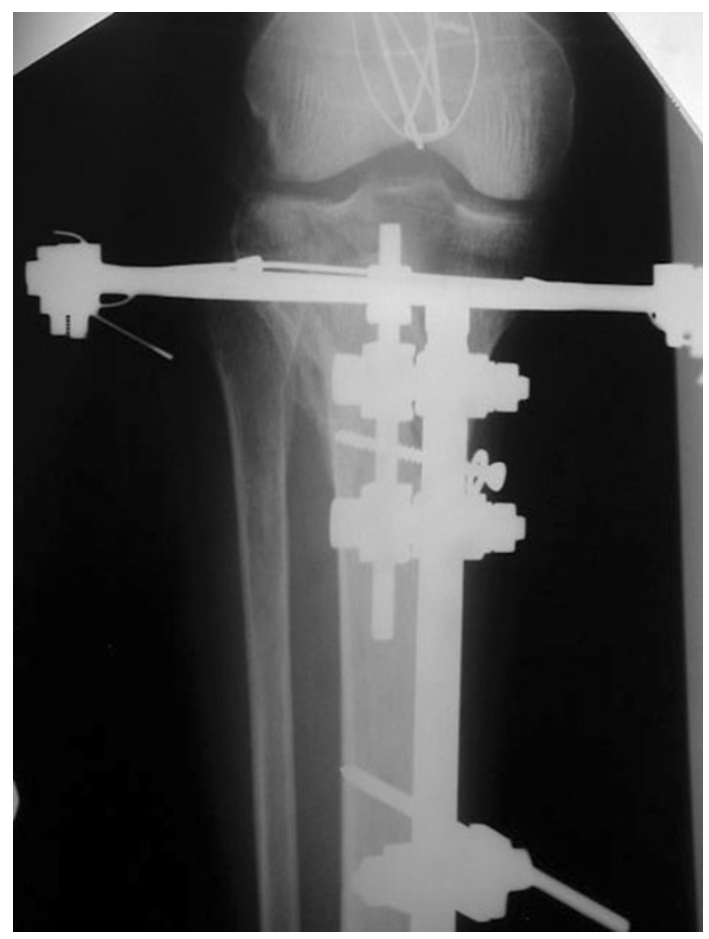

Fig. 6 X-ray post-operative AP view

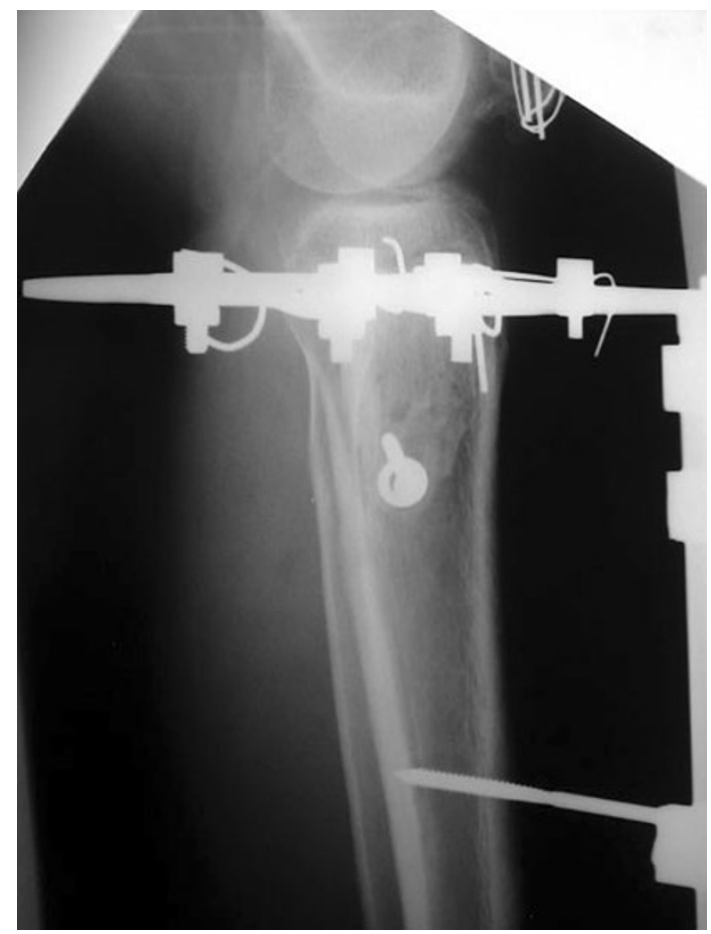

Fig. 7 X-ray post-operative lateral view

Septic arthritis was diagnosed in one patient at 2 weeks post-operatively, arthrotomy and repositioning of wires and a 6-week course of Cefuroxime solved the infection. His functional knee score was 20 at 1 year. Most importantly,

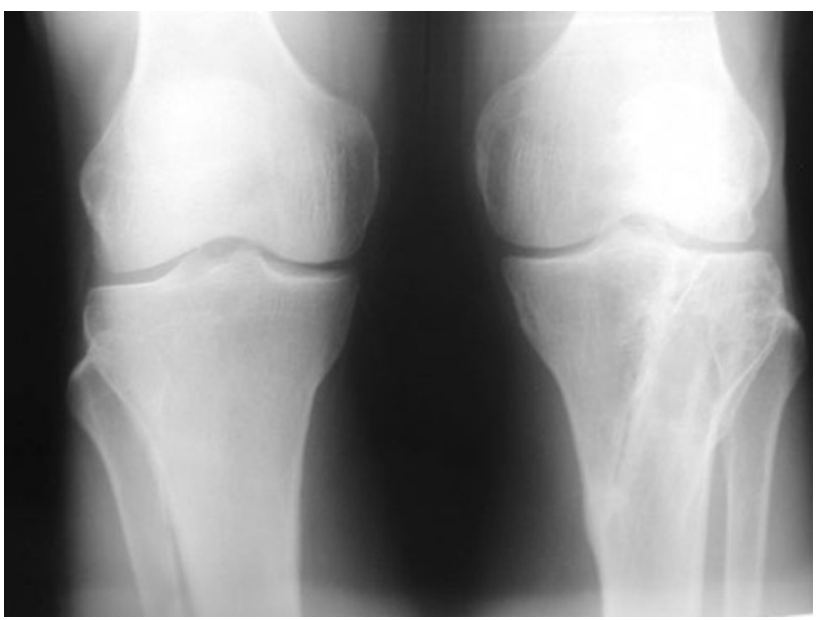

Fig. 8 Comparative knee AP view 2 years post-injury (after removal of prominent patella TBW)

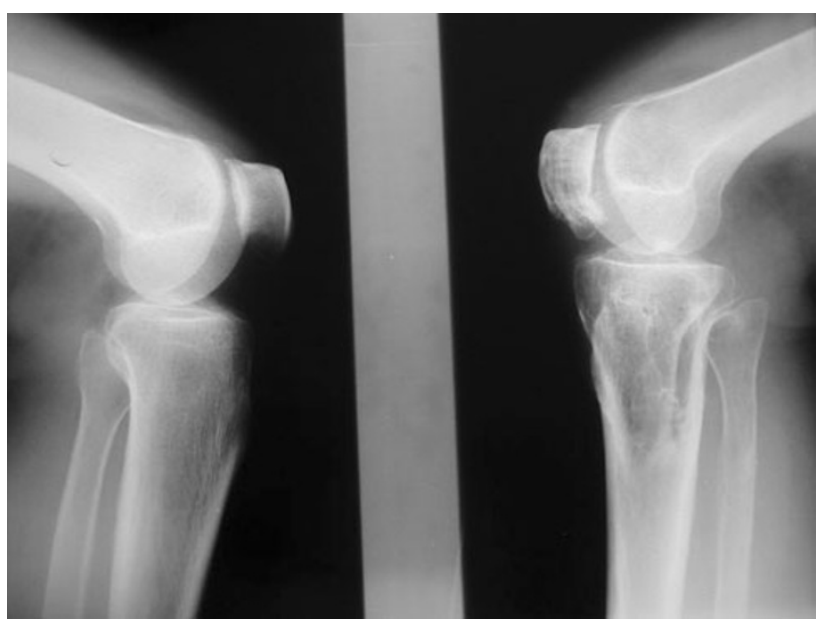

Fig. 9 Comparative knee lateral view 2 years post-injury (after removal of prominent patella $\mathrm{TBW}$ )

there were no cases of soft tissue breakdown or osteomyelitis. None of the patients developed vascular injury or common peroneal nerve injury related to inadvertent Kirschner wire penetration.

Additional surgery was indicated in two patients: one to fix the avulsed PCL at 1 month after the first surgery, and one patient to remove the prominent tension band wires of the patella 6 months after the application of the hybrid frame.

The mean flexion achieved was $120^{\circ}$ (range 90-140 ${ }^{\circ}$ ). Three patients (one with PCL avulsion and two with ipsilateral AO type-C3 supracondylar femoral fracture) had an extension lag of $10-20^{\circ}$. At 12-month follow-up, 15 patients $(48 \%)$ has an excellent Rasmussen knee functional score [10] (27 points or more) (Figs. 10, 11), 13 patients $(42 \%)$ had a good functional (20-26 points), and three patients (10\%) had a fair score (10-19 point). 


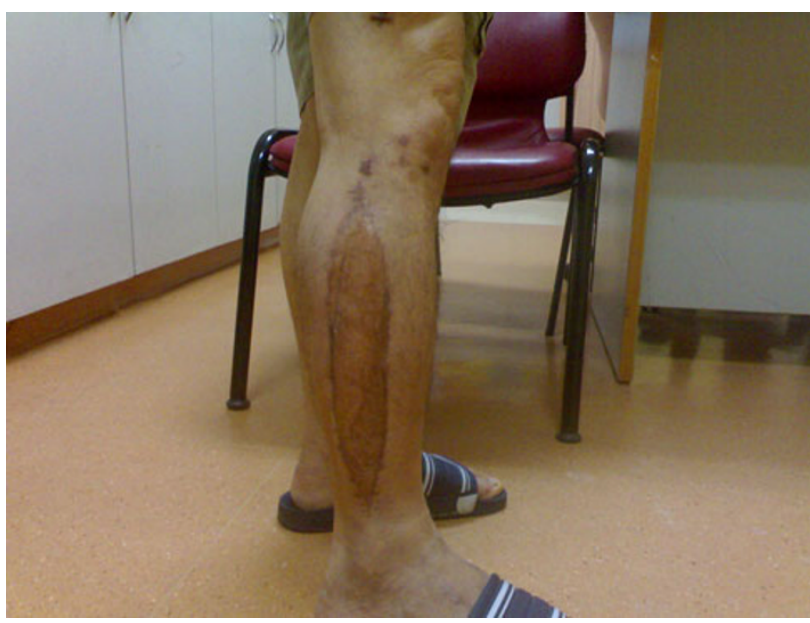

Fig. 10 Knee function: standing at 6 months post-injury

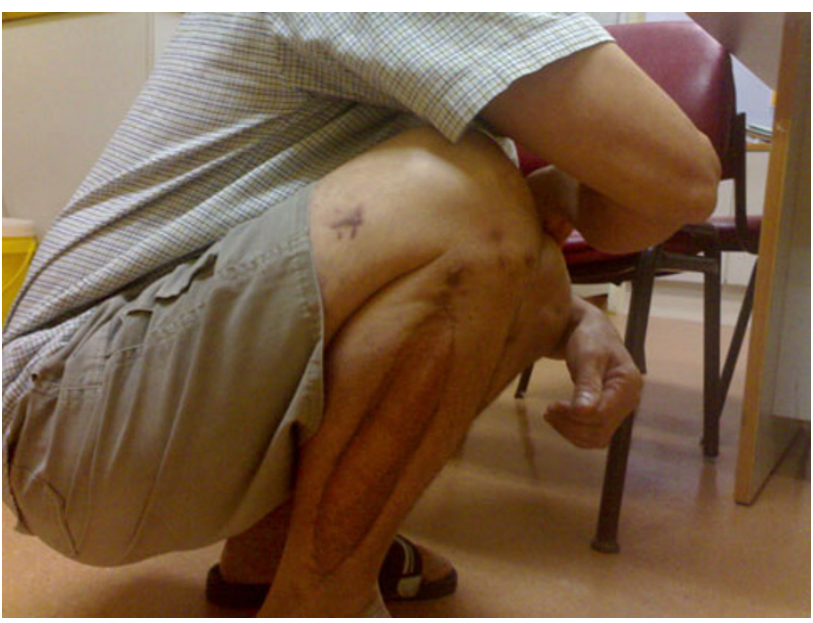

Fig. 11 Knee function: squatting at 6 months post-injury

The fair result was probably attributed to concomitant fractures (two patients have ipsilateral AO type-C3 supracondylar femoral fracture and one has PCL avulsion) (Fig. 11).

\section{Discussion}

In 1979, Schatzker et al. introduced a classification for tibial plateau fractures that distinguished low-energy split depression fractures from higher energy bicondylar (Schatzker V and VI) fractures [11]. Many authors have attempted to define these complex fractures based on the bony injury or the degree of soft tissue injury [12-14]. Watson eloquently defined high-energy tibial plateau fractures based on the presence of large degree of articular depression, displaced multiple condylar fracture lines, and meta-diaphyseal comminution or extension in association with open injuries or extensive soft tissue injury [15]. These injuries were also designated as "severe or complex fractures".

The goals of operative treatment of these fractures include anatomic reduction for restoration of articular congruity and alignment, and stable fixation to allow early motion. However, the classic dual plate osteosynthesis has been associated with potentially devastating complications such as fixation failure, malunion, nonunion, joint stiffness, secondary post-traumatic osteoarthritis, infection, and most importantly severe soft tissue complications ranging from 23 to $87.5 \%$ [1-4, 14].

Over the past few decades, a number of evolving treatment modalities ranging from traction to cast immobilization to open reduction and internal fixation have been used with mixed results. No treatment modality has produced consistently good results, nor has any allowed both stable fixation and preservation of remaining soft tissue [2, 3]. In an attempt to achieve both stable fixation and preserving the remaining soft tissue, many surgeons have chosen to use indirect reduction and external fixation. Hybrid external fixation has been introduced and shown to be effective in the treatment of these difficult fractures [5-8].

Watson et al. applies the term of hybrid fixation quite loosely by stating that hybrid fixation denotes no single or universally accepted treatment strategy or device [16]. The term hybrid has also been used when Schantz pins are mounted on the frame distally for diaphyseal fragment fixation. Kumar and Paige differentiate conventional Ilizarov frame from hybrid Ilizarov frame by using Schantz pins rather than wires through the distal ring for fixation of the diaphysis [17]. With the evolution of technique and hardware used, the current hybrid frames consists of small tensioned wires on a proximal ring frame to maintain and reduce the metaphyseal fragments, while the remainder of the distal frame is attached to the shaft using standard external fixator's half pins [18].

The indications for hybrid external fixation are Schatzker $\mathrm{V}$ and VI tibial plateau fractures, significant metaphyseal comminution with or without diaphyseal extension, severe metaphyseal and subchondral comminution with very small periarticular fragment unsuitable for internal fixation, soft tissue problems like compartment syndrome, open fractures, and severe soft tissue injury in osteoporotic patients [5, 18]. The hybrid external fixator offers several advantages that include minimizing further injury to the soft tissue envelope, feasible surgery even in the presence of badly injured soft tissue, adequate stability to allow early post-operative mobilization, and minimizing risk of stiffness. The disadvantages include the need for constant pin site care, pin sites infection and the risk of septic arthritis from incidental intracapsular pin placement and 
potential risk of common peroneal nerve injury from poor pin placement.

Gaudinez et al. reported the use of Monticelli-Spinelli hybrid frame only for indirect reduction technique in a series of 18 Schatzker V and VI tibial plateau fractures [6]. They recommended the technique due to its advantage of minimal soft tissue complication and allowing early range of motion. In a matched cohort study on the treatment of high-grade tibial plateau fractures comparing internal fixation and ring fixator, Veri et al. [19]. noted high rates of wound complications and reoperation in the open reduction internal fixation utilizing single incision with dual plates group. Their results also suggested that hybrid external fixation is an effective and safe method with a low wound complications rate and early functional return.

Pin tract infection was the main drawback when using an external fixator to treat fractures. In meta-analysis of 10 studies with a total of 381 patients, Hutson et al. encountered 38 cases superficial pin tract infection (10\%), 5 septic arthritis (1\%), and 13 deep infection(4\%) [20]. Our rate of pin tract infection was higher as these pins were inserted on already compromised soft tissue. However, we feel that it is reasonably acceptable to deal with pin tract infection rather than a massive post-operative soft tissue breakdown due to ill-planned incision on already devitalized soft tissue. Septic arthritis is a rare complication arising from use of this technique. It is related to placement of pins or wires in the capsular reflection of the knee joint. This can safely be avoided by placing the proximal most pins or wires $14 \mathrm{~mm}$ below the joint line [9].

\section{Conclusion}

The degree of soft tissue injury associated with tibial plateau fracture is an important determinant for both the choice of treatment modality and the prediction of treatment outcome. Our modified hybrid external fixator is a safe and effective option for the treatment of difficult Schatzker V and VI tibial plateau fractures. It minimizes soft tissue complication and favors bony union with an acceptable return of function. The complications are mainly related to pin tract sepsis. We feel that the technique merits a place in the armamentarium for managing complex tibial plateau fractures.

Open Access This article is distributed under the terms of the Creative Commons Attribution License which permits any use, distribution and reproduction in any medium, provided the original author(s) and source are credited.

\section{References}

1. Young MJ, Barrack RL (1994) Complications of internal fixation of tibial plateau fractures. Orthop Review 23:149-154

2. Schatzker J (1996) Fractures of the tibial plateau. In: Schatzker J, Tile M (eds) The rationale of operative fracture care, 2 nd edn. SpringerVerlag, Berlin, pp 419-438

3. Wiss DA, Watson JT, Johnson EE (1996) Fractures of the knee. In: Rockwood CA, Green DP, Bucholz RW, Heckman JD (eds) Fractures in adults, 4th edn. Lippincott-Raven, Philadelphia New York, pp 1919-1999

4. Moore TM, Patzakis MJ, Harvey JP (1987) Tibial plateau fractures: definition, demographics, treatment rationale, and long results of closed traction management or operative reduction. J Orthop Trauma 1:97-119

5. Stamer DT, Schenk R, Staggers B et al (1994) Bicondylar tibial plateau fractures treated with a hybrid ring external fixator: a preliminary study. J Orthop Trauma 8(6):455-461

6. Gaudinez RF, Mallik AR, Szporn M (1996) Hybrid external fixation of comminuted tibial plateau fractures. Clin Orthop 328:203-210

7. Ali AM, Lang Y, Hashimi M, Saleh M (2001) Bicondylar tibial plateau fractures managed with Sheffield hybrid fixator: biomechanical study and operative technique. Injury 32:86-89

8. Piper KJ, Won HY, Ellis AM (2005) Hybrid external fixation in complex tibial plateau and Plafond fractures: an Australian audit of outcomes. Injury 36:176-184

9. Reid J, Van Slyke M, Moulton M, Mann T (2001) Safe placement of proximal tibial transfixation wires with respect to intracapsular penetration. J Orthop Trauma 15(1):10-17

10. Rasmussen PS (1975) Tibial condylar fractures: Impairment of knee joint stability as indication for surgical treatment. J Bone Joint Surg 55(A): 1331-1350

11. Schatzker J, McBroom R, Bruce D (1979) The tibial plateau fracture: the Toronto experience 1968-1975. Clin Orthop 138:94-104

12. Burri C, Bartzke G, Coldeway J et al (1979) Fractures of the tibial plateau. Clin Orthop 138:84-93

13. Benirschke SK, Agnew SG, Mayo KA et al (1991) Open reduction internal fixation of complex proximal tibial fractures. J Orthop Trauma 5:236

14. Lansinger O, Bergman B, Courmner L et al (1986) Tibial condylar fractures: a 20 year follow-up. J Bone Joint Surg Am 68:13-18

15. Watson TJ (1994) High energy fractures of the tibial plateau. Orthop Clin North Am 25:723-752

16. Watson TJ, Ripple S, Hoshaw SJ, Fyhrie D (2002) Hybrid external fixation for tibial plateau fractures: clinical and biomechanical correlation. Orthop Clin North Am 33:199-209

17. Kumar A, Paige WA (2000) Treatment of complex (Schatzker type VI) fractures of the tibial plateau with circular wire external fixation: retrospective case review. J Orthop Trauma 14:339-344

18. McLaurin TM (2005) Hybrid ring external fixation in the treatment of complex tibial plateau fractures. Tech Knee Surg 4:226-236

19. Veri JP, Blachut P, O’Brien P, Pirani S (2000) High grade tibial plateau fractures: a matched cohort study comparing internal fixation and ring fixator methods. J Orthop Trauma 14:153

20. Hutson JJ Jr, Zych GA (1998) Infections in periarticular fractures of the lower extremity treated with tension wires hybrid fixators. J Orthop Trauma 12:214-218 